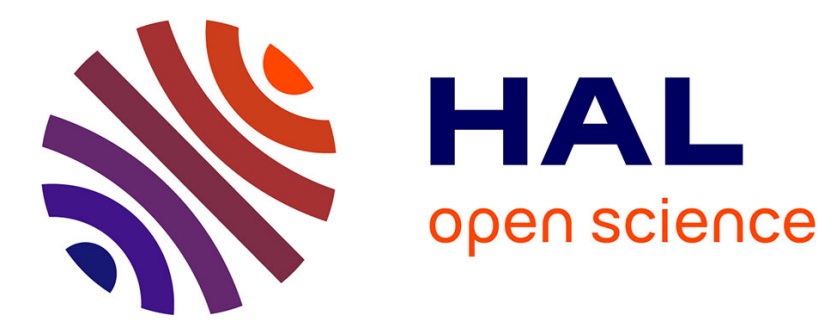

\title{
Do membrane undulations help cells probe the world?
}

Anne Pierres, Virginie Monnet-Corti, Anne-Marie Benoliel, Pierre Bongrand

\section{To cite this version:}

Anne Pierres, Virginie Monnet-Corti, Anne-Marie Benoliel, Pierre Bongrand. Do membrane undulations help cells probe the world?. Trends in Cell Biology, 2009, 19 (9), pp.428-33. 10.1016/j.tcb.2009.05.009 . inserm-00416601

\section{HAL Id: inserm-00416601 https://www.hal.inserm.fr/inserm-00416601}

Submitted on 14 Sep 2009

HAL is a multi-disciplinary open access archive for the deposit and dissemination of scientific research documents, whether they are published or not. The documents may come from teaching and research institutions in France or abroad, or from public or private research centers.
L'archive ouverte pluridisciplinaire HAL, est destinée au dépôt et à la diffusion de documents scientifiques de niveau recherche, publiés ou non, émanant des établissements d'enseignement et de recherche français ou étrangers, des laboratoires publics ou privés. 
(This unedited manuscript was accepted for publication in Trends in Cell Biology and published in volume 19 pp 428-432, 2009)

\title{
Do membrane undulations help cells probe the world?
}

\author{
Anne Pierres ${ }^{1,2}$, Virginie Monnet-Corti ${ }^{1,2,3,4}$, Anne-Marie Benoliel ${ }^{1,2,3}$ and Pierre Bongrand ${ }^{1,2,3,4,5}$. \\ ${ }^{1}$ INSERM UMR600, Lab. Adhesion \& Inflammation, Parc de Luminy, Case 937, 13288 Marseille \\ Cedex 09 France, ${ }^{2}$ CNRS UMR6212, ${ }^{3}$ Université de la Mediterranée, ${ }^{4}$ Assistance Publique-Hôpitaux \\ de Marseille.
}

\begin{abstract}
.
Cells sense physical properties of their environment including substratum rigidity, roughness, and topography of recognition sites. The cell surface displays continuous deformations of nanometer-scale amplitude and $\mathrm{Hz}$ frequency. Recent results support a hypothesis whereby these surface undulations represent a powerful strategy for the rapid acquisition of environmental cues: transient contact with surroundings generate forces of piconewton intensity due to rapid formation and dissociation of intermolecular bonds. The combination of binding and steric forces is expected to drive conformational changes and lateral reorganization of membrane biomolecules, thus generating signaling cascades. We propose that spontaneous membrane mobility shapes the initial information generated by cell-to-surface contacts and thereby biases later consequences of these interactions.
\end{abstract}

\section{Introduction.}

Cells continually take decisions concerning survival, proliferation, differentiation, migration or secretion based upon information relative to the cellular environment. A major challenge for cell biologists is to understand what kind of data cells extract from their environment and how this information is processed to select a particular outcome.

Until recently, the most general explanation for experimental observations is that cells use hundreds of membrane receptors to detect specific ligands either in soluble phase or bound to surfaces. For example integrins detect specific ligands on extracellular matrices, which triggers different responses, ranging from short-term spreading to differentiation [1]. Numerous examples of receptor cross-talk show that cells can integrate responses to a combination of ligands [2]. Finally, cells can respond to the surface density of these ligands as exemplified by the guided migration along haptotactic gradients [3].

In addition to biochemical cues, living cells sense the physical properties of their environment. Many cells need sufficiently rigid substrata to survive, while other cell species preferentially migrate toward stiffer rather than softer surfaces, a phenomenon dubbed durotaxis [4]. Cell differentiation may also be dramatically influenced by surface stiffness: mesenchymal cells deposited on fibronectincoated polymers of varying degree of crosslinking undergo osteoblastic, myoblastic or neuronal differentiation depending on substratum stiffness [5]. Topography is also important, as substrateadherent cells orient along grooves of micrometer width and scale, and were recently found to detect nanoscale topography [6,7]. The macrophage capacity to phagocytize is dependent on particle shape [8]. The homogeneity and spacing of specific ligands and membrane receptors can also influence cell behaviour, murine fibroblast migration on surfaces coated with integrin ligands require a minimum amount of clustering [9] and efficient spreading require ligand spacing of less than 50-100 $\mathrm{nm} \mathrm{[10].} \mathrm{T}$ lymphocyte activation on surfaces coated with nanopatterned stimulatory molecules are also influenced by topography [11].

\footnotetext{
${ }^{5}$ Corresponding author, Email pierre.bongrand@inserm.fr
} 
One way of identifying the information perceived by a cell approaching a surface consists of examining the sequence of events generated by this interaction at a molecular scale, within the first seconds of contact.

Cell membranes display continuous deformations on a wide range of timescales.

The molecular interactions generated by cell-to-surface approach are shaped by membrane dynamics. Numerous reports yield a detailed description of typical deformation patterns on a timescale of several tens of seconds. Several interesting reports also demonstrate the occurrence of rapid membrane undulations in various cell types.

"Conventional" membrane deformation (10s-1minute time-scale).

Cell membranes continously generate different kinds of protrusions. Recent data have improved our understanding of the physical properties of each type of protrusion.

Finger-like structures such as filopodia or microvilli are cylindrical protrusions with a typical diameter of 0.1-0.2 $\mu \mathrm{m}$ and a length ranging between a few tenths of a micrometer and several micrometers [12]. Their core is made of a bundle of typically 10-30 actin filaments and they have often been ascribed an exploratory function [13] due to the presence of membrane receptors on their tip and continuous protrusion-retraction cycles. The characteristic timescale of these cycles is on the order of tens of seconds [14]. The characteristic pushing force they may resist to is estimated at several tens of piconewtons [15], with a traction force on the order of $10 \mathrm{pN} \mathrm{[16].}$

Sheet-like structures such as lamellipodia have a typical thickness of 100-200 nm [12] and several micrometer width. The leading edge is underlined with a network of branched microfilaments. Lamellipodia display protrusion and retraction phases with a duration of several tens of seconds [17]. The force required to stall or markedly reduce lamellipodial expansion is estimated at a few tens of piconewtons per micrometer of leading edge $[18,19]$.

Blebbing. In addition to aforementioned modes of cell deformation, cell membranes can display transient bulges that were interpreted as a consequence of detachment of the plasma membrane from underlying cortical microfilaments and existence of an intracellular pressure in excess of the outer medium pressure [20]. Blebs may protrude up to $2 \mu \mathrm{m}$ height and they display a cycle of rapid expansion followed by a slower retraction, on the time-scale of 1 minute. While blebbing has often been ascribed to some sort of cellular damage, it may be involved in nonapoptotic cell migration and tissue invasion by cancer cells [20].

\section{Rapid membrane undulations (1 second timescale).}

While the three modes of cell deformation we have just described are familiar to cell biologists, their characteristic time-scale of several tens of seconds may not be sufficient per se to allow cells to fathom their environment with sufficient rapidity. Pathogen ingestion by blood neutrophils is completed within a few seconds [21] and the arrest of flowing leukocytes on inflamed endothelia involves subsecond activation of their membrane integrins [22]. A clue to solving this difficulty may come from reports demonstrating the existence of transverse membrane undulations with a frequency of $1 \mathrm{~Hz}$ or more. While the erythrocyte membrane displays high frequency undulations (the frequency of this « flickering » could be as high as $1,000 \mathrm{~Hz}$ with an amplitude ranging between a few tens and hundreds of nanometers [23,24]), plasma membrane undulations of nucleated cells were first thought to be absent because of excessive surface tension and bending rigidity (Box 1). However, it was later shown using dark field illumination [25] that blood lymphocytes or monocytes displayed transverse surface undulations with $0.2-30 \mathrm{~Hz}$ frequency, and 20-30 nm amplitude. More recently, monitoring the surface of human [26] or murine [27] fibroblasts with atomic force microscopy revealed force fluctuations with a frequency between 0.5 and $0.01 \mathrm{~Hz}$ and amplitude between 20 and $80 \mathrm{pN}$, corresponding to $1-4 \mathrm{~nm}$ displacement. Interestingly, these undulations were not impaired by drugs depolymerizing microfilaments or microtubules, in accordance with a passive Helfrich-type mechanism (Box 1).

Atomic force microscopy gives direct information on the opposing face of adherent cells, while interference reflection microscopy / reflection interference contrast microscopy (IRM / RICM) allows direct monitoring of the underside of cells approaching a surface. Murine $\mathrm{J} 774$ macrophages [28] displayed undulations of about $5 \mathrm{~nm}$ amplitude. This amplitude was increased twofold when cells 
were treated with latrunculin for microfilament inhibition, thus decreasing membrane bending rigidity. Interestingly, measured parameters were consistent with predictions from Helfrich theory about passive thermal oscillations (Box1). Human THP-1 monocytic cells deposited on fibronectin-coated surfaces [29] display transverse fluctuations with an amplitude of 2-10 $\mathrm{nm}$ in the $\mathrm{Hz}$ frequency range. Some discrepancies with predictions from Helfrich theory suggested an active cell participation: while the undulation amplitude of a passive sheet is expected to decrease when it approaches a surface, this expected behaviour was found when THP-1 cells were separated from the surface by more than about $60 \mathrm{~nm}$, but amplitude increased when the distance was further decreased. Also, short-distance undulations increased 2-3 minutes after contact initiation. Thus, cells might perceive an approaching surface at $60 \mathrm{~nm}$ distance.

In conclusion living cells display continual membrane undulations with a time-scale of 1-10s. These movements can generate forces of several tens of piconewtons. Our hypothesis is that these deformations have the capacity to initiate and modulate substantial signaling events occurring during the initial cell-surface contact. This is supported by a number of recent reports aimed at determining the influence of piconewton forces on signaling cascades. It is therefore important to assess evidence that these forces can initiate or modulate known mechanisms of signal generation.

Box 1. A theoretical framework for analyzing spontaneous membrane undulations. It has been amply demonstrated that "passive" membranes such as envelopes of large unilamellar vesicles or erythrocytes displayed spontaneous undulations [23,76]. Recently, the membranes of eukaryotic cells were also found to display continuous pulsating deformations [25-29]. The theoretical model built by Helfrich [77] remains the main basis of current thinking [76]: a flexible and flaccid sheet may undergo a number of conformations involving the formation of various bulges. If the boundaries of the sheet are fixed, any departure from the simplest planar shape will involve an increase of total area and curvature. This will result in energy increase proportional to the surface tension $\sigma$ and bending rigidity $\kappa$. A quantitative way of dealing with these deformations relies on Fourier analysis. Any shape is described as a combination of sinusoïdal deformations. The contribution of each sinusoidal deformation is estimated on the basis of Boltzmann's law. Thus, the mean squared amplitude of a deformation of wavelength $\lambda$ is estimated at $k_{B} T / A\left(\sigma q^{2}+\kappa q^{4}\right)$, where $\mathrm{k}_{\mathrm{B}}$ is Boltzmann's constant, $\mathrm{T}$ is the absolute temperature, $\mathrm{A}$ is the sheet area, and $\mathrm{q}=2 \pi / \lambda$. $\mathrm{A}$ noticeable consequence of this model is that these undulations should generate a repulsive pressure on the order of $\left(\mathrm{k}_{\mathrm{B}} \mathrm{T}\right)^{2} / \mathrm{Kd}^{3}$ between surfaces separated by a distance $\mathrm{d}$, thus inhibiting adhesive interactions. However, it was also emphasized that these undulations might favor local encounters between ligands and receptors, even if the average separation between surfaces was wider than the size of ligands and receptors, due to the presence of bulky repellers [28]. A last point is that it was long thought that the surface tension and bending rigidity of nucleated cells were too high to allow significant undulations, following aforementioned Helfrich formula. However, when a quantitative analysis of membrane shape and fluctuations near a surface was used to derive numerical estimates for the surface tension and bending rigidity, estimates of $10^{-4} \mathrm{~N} / \mathrm{m}$ and about $1,000 \mathrm{kBT}$ were obtained for monocytic cell lines. While the bending rigidity was at least twentyfold higher than that of red blood cells or lipid bilayers, this was shown to be consistent with the occurence of passive, thermal nanometer-scale undulations [28], although the involvement of other active mechanisms could not be ruled out.

\section{Forces generated by membrane undulations can influence early signaling cascades triggered by membrane receptors.}

The engagement of cell membrane receptors usually triggers a complex set of intracellular events resulting from the multiple occurrence of two basic molecular processes: a) The structure of a given molecule may be altered, either through a conformational change or by chemical modification of a surface group, e.g. tyrosine phosphorylation. This may result in exposition of new recognition sites or enzymatic site activation. The simplest case may be the opening of a membrane-embedded ionic channel [30]. b) Two molecules may bind to each other, which requires both the exposition of new recognition sites and that they encounter each other. The importance of the latter condition must be considered in view of the high number of potential interactions between biomolecules: over 250,000 interactions between about 22,000 proteins were compiled in the Unified Human Interactome database [31]. A remarkable example is the formation of focal adhesions : these molecular scaffolds involve over 150 molecular species and may appear a few minutes after integrin-mediated adhesion between cells and cellular matrices [32]. As described in several recent reviews [33,34], focal adhesions 
respond to forces of several tens to hundreds of piconewton by contact extension and signaling events such as tyrosine phosphorylation, small $\mathrm{G}$ protein activation or intracellular calcium rise. Cadherinmediated adhesions share similar properties [35]. However, other evidence described below suggests that piconewton forces applied during a few second to a single molecule on the cell membrane can generate significant signals.

i) Conformation. While a ligand with a highly specific molecular shape may allosterically change receptor conformation [36], mechanical forces can also alter the conformation of different molecules. The mechanosensitive MscL channel found in $E$ coli membrane can be opened with a surface tension on the order of 0.01 newton/m. Forces applied to extracellular matrices by cultured myofibroblasts were found to expose active mediators such as TGFbeta [37]. A force of $2 \mathrm{pN}$ may partially unfold the integrin-linked talin molecule, thus disclosing vinculin binding sites [38]. Recent studies suggest that piconewton forces can facilitate a subsecond conformational change of leukocyte integrins interacting with endothelial cells under flow [39]. These findings were often interpreted as a direct consequence of Boltzmann's law [40]. A force is expected to influence a conformational change if the product of the force (in piconewtons) times the displacement (in nanometers) is comparable to or higher than $\approx 4$. A force of $10 \mathrm{pN}$ generated by membrane pulsations [26] may thus significantly alter the frequency of a conformational change involving a molecular deformation of order of $0.7 \mathrm{~nm}$ (Fig. 1B) such as were reported in recent studies of integrin structure [41].

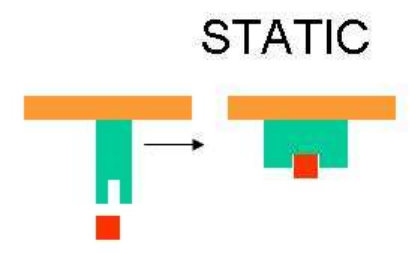

A

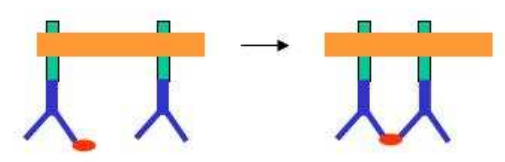

B

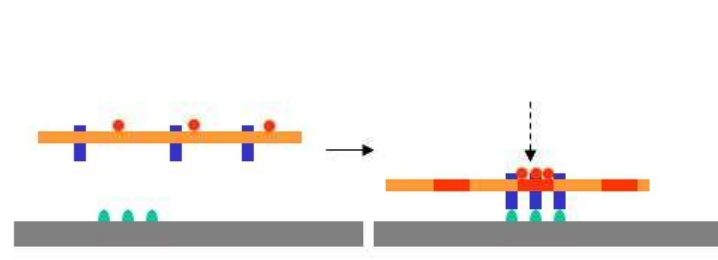

C

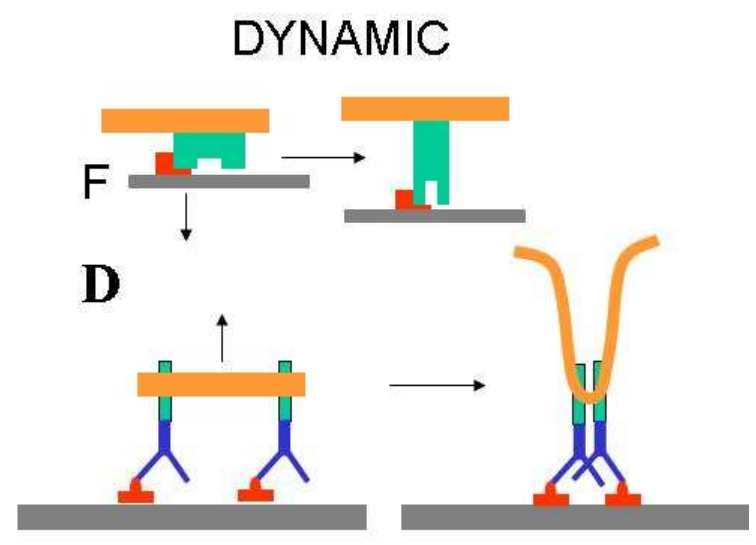

$\mathbf{E}$

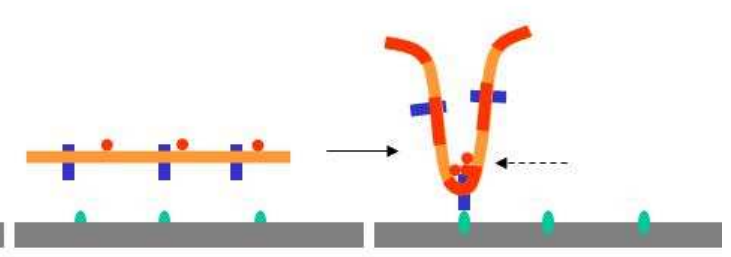

$\mathbf{F}$

Figure 1. Expected influence of membrane dynamics on signaling. The interaction of a static receptorbearing membrane and free or attached ligands may generate signaling cascades through a combination of at least three mechanisms: A receptor conformational change may expose activating sites or open ionic chanels, $\mathbf{B}$ receptor cross-linking by a ligand cluster may result in mutual interaction between intracytoplasmic molecules, C active sites may nucleate clusters of intracytoplasmic molecules and/or membrane microdomains (broken arrow) resulting in signalosome formation.

Membrane fluctuations may initiate similar phenomena: D Pulling at a membrane receptors may generate/stabilize a new conformation and increase the lifetime of interaction with their ligands if they form catch bonds . E Membrane retraction may result in clustering of attached molecules on the tip of a protrusion. $\mathbf{F}$ Membrane local curvature as a consequence of shape fluctuations or pulling forces may result in local clustering of membrane associated molecules. 
ii) Clustering of membrane-associated molecules. Another major mechanism of signal generation involves receptor clustering or even multimerization [42]. Many receptors are associated with a tyrosine kinase. Facilitating encounters between a kinase and its target tyrosine will allow phosphorylation and recruitement of adapter proteins (containing SH2 or PTB domains specific for the newly phosphorylated tyrosine) thus leading to the formation of a multimolecular signaling complex. While ligand binding can trigger receptor dimerization [42], the retraction of a membrane region exposing several receptors bound to a surface might concentrate receptors in the shrinking contact zone (Fig. 1D). Indeed, when lipid vesicles bound to planar surfaces through mobile integrins were subjected to a vertical pull of a few piconewtons, integrin concentration increased in the contact area [43]. Similar results were reported when disruptive forces were exerted on conjugates made between cytotoxic lymphocytes and target cells [44].

iii) Formation of multimolecular signaling complexes ("signalosomes"). Signal generation might be dramatically facilitated by the formation of microdomains concentrating desirable molecules. This is best illustrated by a recently suggested model for $\mathrm{T}$ lymphocyte activation [45]. When a T lymphocyte encountered ligands of its specific receptor (TCR) on an antigen-presenting cells, a region of tight intercellular contact is formed, with local trapping of TCRs (that are retained by specific interactions with their antigen) and steric exclusion of some membrane associated phosphatases with bulky extracellular domains. This might change the balance between phosphorylation and dephosphorylation events, thus triggering a signaling cascade. Interestingly, a number of recent theoretical and experimental studies demonstrate that the kinetic, mechanical and geometrical changes imposed on cell-surface contact by membrane undulations have the capacity to influence the lateral redistribution of membrane molecules through the following mechanisms:

a) Steric effects. The formation of a region of tight contact between membranes and other surfaces may require the exclusion of bulky molecules otherwise responsible for steric stabilization. Membrane undulations might influence this redistribution in three different ways:

- First, intermembrane repulsion depends on the kinetics of contact formation [46,47].

- Second, local protrusions resulting from undulations may overcome a repulsive barrier [28].

- Third, transverse membrane undulations should greatly enhance lateral redistribution of membrane molecules [48]. The potential importance of enhancing lateral diffusion of membrane molecules is supported by the (opposite) finding that signaling could be strongly altered by impairing lateral mobility of membrane molecules with nanobarriers [49].

b) Membrane curvature. An intriguing possibility would be that the lateral reorganization of membrane molecules be influenced by the curvature changes related to membrane deformation. First, membrane lipids may have a particular affinity for regions whose curvature matches the size and shape of hydrophobic tails. Second, intrinsic membrane proteins may be excluded from or attracted by high curvature regions [50]. Third, intracellular molecules with a potential signaling activity could bind to membrane regions with a particular curvature [51] through specific interactions between molecular domains of these molecules and membrane regions with a matching curvature. It is therefore interesting to recall that forces of several tens of piconewtons imposed with micropipette aspiration can readily alter membrane curvature [52], and, more recently, similar forces applied through adhesive points were found to form membrane tubes [53]. Similar deformations may thus be generated by undulations of membraned pinned to solid surfaces through ligand-receptor interactions.

An important point is that the forces generated by interactions between undulating membranes and foreign surfaces are dependent on molecular properties of ligand-receptor interactions that have long been ignored when molecular interactions were studied with conventional methods (Box 2). Firstly, the forces that can be sustained by bonds formed between surfaces and undulating membranes are incompletely correlated to binding affinity [54]. Secondly, recently evidenced catch bonds, the lifetime of which is increased by forces [55-57], may be revealed by dynamic interactions between a fluctuating membrane and a surfaces. Thirdly, recent results [58] suggest that bond formation between two mobile surfaces bearing adhesion molecules requires a minimum contact time, thus, transient interactions between membranes may select a few receptors with particular binding parameters, length and flexibility out of a number of potential interactions. 
Box 2. Molecular interactions between surface-attached molecule. A new theoretical framework. Conventional studies made on interactions between soluble molecules led to a fairly simple conceptual framework that has been considered fairly satisfactory for decades [78]: two molecules A and B may be clearly viewed as bound or separated. The probability that two molecules A and B bumping again each other will form a complex is simply related to the kinetic association constant $\mathrm{k}_{\text {on }}$. The rate of spontaneous dissociation of a complex is proportional to the kinetic dissociation rate $\mathrm{k}_{\text {off. }}$. Thus, two simple parameters are sufficient to account for all interactions between molecules A and B. Recently, this conventional framework was found insufficient to account for interactions between surfaceattached molecules, based on experimental studies performed on single ligand-receptor pairs [78]. The dissociation rate of a complex is modified when a disruptive force is applied. In many cases, this modification follows Bell's law $: \mathrm{k}_{\text {off }}=\mathrm{k}_{\text {off }}(0) \exp \left(\mathrm{F} / \mathrm{F}^{\circ}\right)$, where $\mathrm{F}^{\circ}$ ranges between several piconewtons and several tens of piconewtons. In several cases, a pulling force was even shown to increase the lifetime of molecular bonds that were dubbed "catch bonds"

[55-57]. The association or separation of molecules A and B follows a complex reaction path with multiple intermediate binding steps and barriers. The property of a complex thus depends on its history that determines the number of barriers that were passed after initial ligand-receptor association [79-80]. The association rate of surfaceattached molecules is highly dependent on the distance between surfaces, and the length or flexibility of the linker

between binding sites and surfaces. It is difficult to relate the quantitative properties of multivalent attachment

between surfaces to single bond properties due to geometrical and rebinding effects. As a consequence, the probability of bond formation between an undulating membrane and a foreign surface cannot be derived from the densities of ligands and receptors, and kinetic parameters $\mathrm{k}_{\mathrm{on}}$ and $\mathrm{k}_{\mathrm{off}}$ that would be intrinsic properties of binding sites. Rather, the forces generated between undulating membranes and surfaces is dependent on complex functions

$\mathrm{k}_{\text {off }}(\mathrm{F})$ (i.e. dissociation rate as a function of applied force) and $\mathrm{k}_{\mathrm{on}}(\mathrm{d})$ (i.e. association rate as a function of intermolecular distance) that depend on the structure of binding sites, length and flexibility of membrane receptors, and properties of surrounding molecules.

All aforementioned data and models are a strong incentive to further explore the hypothesis that early signals generated by cell interactions with foreign surfaces are shaped by dynamic contacts whose properties are a consequence of membrane dynamics. Many experimental tools are currently available to explore this possibility. A reasonable strategy would consist of altering membrane undulations by modifying membrane tension (as might be achieved with inhibitors of myosin or small $\mathrm{G}$ proteins) or bending rigidity (e.g. by acting on lipid composition or underlying microfilaments), or linkage between the plasma membrane and underlying cytoskeleton [59]. Early intracellular signals can now detected by an increasing number of tools such as fluorescent calcium probes, FRET-based reporters of kinase [60-61] or G-protein [62] activity, or redistribution of fluorescent fusion proteins [63]. The major problem will be to make sense of experimental data and improve our understanding of cell response to signals. It is abundantly clear that new theoretical tools will be needed to interpret the growing amount of experimental results.

\section{The next challenge will be to understand how cells process entering information flow modulated by membrane undulations to take decisions.}

While it is well recognized that the initial cell-cell or cell-substratum interaction and mechanosensing events occur on the subsecond to second timescale [64], the current challenge consists firstly of detecting, and secondly of interpreting these events. Building a coherent framework to achieve this interpretation will require the reexamination of basic terms and the address of simple questions as follows :

i) what is the meaning of currently used words such as cell decision [65], cell state [65] or cell fate [66] ? Indeed, after identifying a sequence of early intracellular biochemical events following cell encounter with a surface, it is important to determine to what extent and how long these events will influence cell behaviour. Clearly, the view of a rugged epigenetic landscape with valleys representing preprogrammed attractor states [67] may provide an interesting way of formulating the underlying concept and deserves being analyzed.

ii) What is a signal, which may be considered as a unit message considered by cells to take a decision ? This is a key question to take advantage of information theory, whose relevance to biological systems was plagued by the recognized difficulty of defining information in this domain, an enterprise that was deemed "difficult and often contentious" [68], with a possible exception for genetics and neuroscience. An interesting formulation would be a local production of second messengers or 
enzyme activation, leading to the spatial segregation of signal flow and formation of microdomains of activated signaling components [69]

iii) when these concepts have been clearly defined, there will remain to determine how signals are processed by cells, which already generated interesting problems : a) is signal processing local or global ? in view of somewhat opposite results [70-72], it is likely that different signals may behave in opposite ways. b) what is the duration of the period of time used for signal integration ? time integration is indeed necessary to deal with noise, and it was recognized as a requirement to achieve excellent spatial accuracy [34]. A related problem is the elucidation of mechanisms used by cells to account for signal duration [73] and decode signal frequency [74]. c) Are cells endowed with specific means of disregarding undesirable noise [75].

Conclusion. Cell membranes display continuous deformations with a timescale ranging between less than a second and several seconds. These deformations have the capacity to strongly influence the signals generated by cell interaction with foreign surfaces. We suggest that it would be highly rewarding i) to record as exhaustively as possible the earliest signals generated by cell-surface interactions, ii) to look for causal relationships between these signals and cell membrane movements, and iii) to analyze the way these signals are processed. In addition to a general theoretical interest, a better understanding of cell reaction at interfaces should be of practical use in different domains such as biomaterial design.

Acknowledgment. Part of the work described in this review was supported by ARC. The authors thank Dr. Annemarie Lellouch for a critical reading of the manuscript.

\section{References.}

1 Miranti, C. K. and Brugge, J. S. (2002) Sensing the environment: a historical perspective on integrin signal transduction. Nature Cell Biol. 4, 83-90.

2 Morgan, M. R.et al. (2007) Synergistic control of cell adhesion by integrins and syndecans. Nature Rev. Mol. Cell Biol. 8, 957-969.

3 Rhoads, D. S. and Guan, J-L. (2007) Analysis of cell migration on defined fibronectin gradients: role of intracellular signaling molecules. Exp. Cell Res. 313, 3859-3867.

4 Lo, C. M. et al. (2000) Cell movement is guided by the rigidity of the substrate. Biophys. J. 79, 144-152.

5 Engler, A. J. et al. (2006) Matric elasticity directs stem cell lineage specification. Cell 126, 677-689.

6 Dalby M. J. et al. (2006) Osteoprogenitor response to defined topographies with nanoscale depths. Biomaterials 27, 1306-1315.

7 Dalby M. J. et al. (2007) The control of human mesenchymal cell differentiation using nanoscale symmetry and disorder. Nature Materials 6 997-1003.

8 Champion, J. A. and Mitragori, S. (2006) Role of target geometry in phagocytosis. Proc. Natl. Acad. Sci. USA 103, 4930-4934.

9 Maheswari, G. et al. (2000) Cell adhesion and motility depend on nanoscale RGD clustering. J. Cell Sci. 113, 1677-1686.

10 Calvacanti-Adam, E. A. et al. (2007) Cell spreading and focal adhesion dynamics are regulated by spacing of integrin ligands. Biophys. J. 92, 2964-2974.

11 Shen, K. et al. (2008) Micropatterning of costimulatory ligands enhances CD4 T cell functions. Proc. Natl. Acad. Sci. USA 105, 7791-7796.

12 Chhabra, E. S. and Higgs, H. N. (2007) the many faces of actin: matching assembly factors with cellular structures. Nature Cell Biol. 9, 1110-1121.

13 Faix, J. and Rottner, K. (2006) The making of filopodia. Current Opinion Cell Biol. 18, 18-25.

14 Baker, M. W. and Macagno, E. R. (2007) In vivo imaging of growth cone and filopodial dynamics: evidence for contact-mediated retraction of filopodia leading to the tiling of sibling processes. J. Comp. Neurol. 500, 850862.

15 Mogilner, A. and Rubinstein, B. (2005) The physics of filopodial protrusion. Biophys. J. 89, $782-795$.

16 Kress, H. et al. (2007) Filopodia act as phagocytic tentacles and pull with discrete steps and a load-dependent velocity. Proc. Natl. Acad. Sci. USA 104, 11633-11638.

17 Dubin-Thaler, B. J. et al. (2004) Nanometer analysis of cell spreading on matrix-coated surfaces reveals to distinct cell states and STEPs. Biophys. J. 86, 1794-1806.

18 Bohnet, S. et al. (2006) Weak force stalls protrusion at the leading edge of the lamellipodium. Biophys. J. 90, 1810-1820. 
19 Prass, M. et al. (2006) Direct measurement of the lamellipodial protrusive force in a migrating cell. J. Cell Biol. 174, 767-772.

20 Fackler, O. T. and Grosse, R. (2008) Cell motility through plasma membrane blebbing. J. Cell Biol. 181, 879884.

21 Evans, E. (1989) Kinetics of granulocyte phagocytosis: rate limited by cytoplasmic viscosity and constrained by cell size. Cell. Motil. Cytoskeleton 14, 544-551.

22 Shamri, R. et al. (2005) Lymphocyte arrest requires instantaneous induction of an extended LFA-1 conformation mediated by endothelium-bound chemokines. Nature Immunol. 6, 497-606.

23 Rappaz, B. et al. (2009) Spatial analysis of erythrocyte membrane fluctuations by digital holographic microscopy. Blood Cells Mol. Dis. doi:10.1016/j.bemd.2009.01.018.

24 Evans, J. et al. (2008) Fluctuations of the red blood cell membrane: relation to mechanical properties and lack of ATP dependence. Biophys. J. 94, 4134-4144.

$25 \mathrm{Krol}$, A. Y. et al. (1990) Local mechanical oscillations of the cell surface within the range 0.2-30 Hz. Eur. Biophys. J. 19, 93-99.

26 Pelling, A. E. et al. (2007) Mapping correlated membrane pulsations and fluctuations in human cells. J. Mol Recognition 20, 467-475.

27 Szabo, B. et al. (2002) Atomic force microscopy of height fluctuations of fibroblast cells. Phys. Rev. E 65:041910.

28 Zidovska, A. and Sackmann, E. (2006) Brownian motion of nucleated cell envelopes impedes adhesion. Phys. Rev. Letters 96:048103.

29 Pierres, A. et al. (2008) How cells tiptoe on adhesive surfaces before sticking. Biophys. J. 94:4114-4122.

30 Chalfie, M. (2009) Neurosensory mechanotransduction. Nature Rev. Mol. Cell Biol. 10:44-52.

31 Chaurasia, G. et al. (2008) UniHI 4 : new tools for query, analysis and visualization of the human proteinprotein interactome. Nucleic acids research 1-4.

32 Zaidel-Bar, R. et al. (2007) Functional atlas of the integrin adhesome. Nature Cell Biol. 9:858-867.

33 Bershadsky, A., Kozlov, M. and Geiger, B. (2006) Adhesion-mediated mechanosensitivity: a time to experiment and a time to theorize. Current Opinion Cell Biol. 18:472-281.

34 Geiger, D., Spatz, J. P. and Bershadsky, A. D. (2009) Environmental sensing through focal adhesion. Nature Rev. Mol. Cell Biol. 10:21-33.

35 Schwartz, M. A. and DeSimone, D. W. (2008) Cell adhesion receptors in mechanotranduction. Current Opinion Cell Biol. 20, 551-556.

36 Rosenbaum, D. M. et al. (2007) GPCR engineering yields high-resolution structural insights into beta2adrenergic receptor function. Science 318, 1266-1273.

37 Wipff, P-J. and Hinz, B. (2008) Integrins and the activation of latent transforming growth factor beta 1 - An intimate relationship. Eur. J. Cell Biol. 87:601-615.

38 Del Rio, A. et al. (2009) Stretching single talin rod molecules activates vinculing binding. Science 323, 638641.

39 Alon, R. and Dustin, M. L. (2007) Force as facilitator of integrin conformational changes during leukocyte arrest on blood vessels and antigen-presenting cells. Immunity 26:17-27.

40 Landau, L. and Lifchitz, E. (1967) Physique Statistique, Mir Publisher, Moscow, p135.

41 Astrof, N. S. et al. (2006) Importance of force linkage in mechanochemistry of adhesion receptors. Biochemistry 45, 15020-15028.

42 Yang, Y. et al. (2008) Contacts between membrane proximal regions of the PDGF receptor ectodomain are required for receptor activation but not for receptor dimerization. Proc. Natl. Acad. Sci. USA 105, 7681-7686.

43 Smith, A. S. et al. (2008) Force-induced growth of adhesion domains is controlled by receptor mobility. Proc. Natl. Acad. Sci. USA 105:6906-6911.

44 Tozeren, A., Sung, K. L. P. and Chien, S. (1989) Theoretical and experimental studies on cross-bridge migration during cell disaggregation. Biophys. J. 55:479-487.

45 Burroughs, N. G. et al. (2006) Ligand detection and discrimination by spatial relocalization: a kinasephosphatase segregation model of TCR activation. Biophys. J. 91, 1619-1629.

46 Patel K. D. et al. (1995). P-selectin must extend a sufficient length from the plasma membrane to mediate rolling of neutrophils, J. Cell Biol., 131, 1893-1902.

47 Sabri S. et al.. (2000) Glycocalyx modulation is a physiological means of regulating cell adhesion. J. Cell Sci. $113,1589-1600$.

48 Brown, F. L. H. (2003) Regulation of protein mobility via thermal membrane undulations. Biophys. J. 84, $842-853$.

49 Mossman, K. D. et al.. (2005) Altered TCR signaling from geometrically repatterned immunological synapses. Science 310, 1191-1193.

50 Reynwar, B. J. et al. (2007) Aggregation and vesiculation of membrane proteins by curvature-mediated interactions. Nature 447, 461-464. 
51 Suetsugu, S. et al. (2006) The RAC binding domain/IRSP53-MIM homology domain of IRSp53 induces RAC-dependent membrane deformation. J. Biol. Chem. 281, 35347-35358.

52 Herant, M., Heinrich, V. and Dembo, M. (2005) Mechanics of neutrophil phagocytosis: behavior of cortical tension. J. Cell Sci. 118:1789-1797.

53 Tabdanov, E. et al. (2009) Role of E-cadherin in membrane-cortex interaction probed by nanotube extrusion. Biophys. J. 96:2457-2465.

54 Pierres, A. et al. (2006) Dissecting individual ligand-receptor bonds with a laminar flow chamber. Biophys. Rev. Lett. 1: 231-257.

55 Dembo, M. et al. (1988) The reaction-limited kinetics of membrane-to-surface adhesion and detachment. Proc. Roy. Soc. Lond. B 234, 55-83.

56 Thomas, W. E et al. (2002) Bacterial adhesion to target cells enhanced by shear forces. Cell 109, 913-923.

57 Marshall, B. et al. (2003) Direct observation of catch bonds involving cell-adhesion molecules. Nature 423, 190-193.

58 Robert, P. et al. (2009) Biomolecule association rates do not provide a complete description of bond formation. Biophys. $J$. in press.

59 Raucher, D. (2000) Phosphatidylinositol 4, 5 Bisphosphate functions as a second messenger that regulates cytoskeleton-plasma membrane interaction. Cell 100:221-228.

60 Wang, Y. et al. Visualizing the mechanical activation of Src. Nature 434:1040-1045.

61 Randriamampita, C. (2008) A novel ZAP-70 dependent FRET based biosensor reveals kinase activity at both the immunological synapse and the antisynapse. PloS ONE 3:e1521.

62 Pertz, O. (2006) Spatiotemporal dynamics of RhoA activity in migrating cells. Nature 440:1069-1072.

63 Charras, G. T. (2006) Reassembly of cortical actin in cell blebs. J. Cell Biol. 175:477-490.

64 Vogel, V. and Sheetz, M. (2006) Local force and geometry sensing regulate cell functions. Nature Rev. Mol. Cell Biol. 7:265-275.

65 Koseska, A. et al. (2009) Timing cellular decision making under noise via cell-cell communication. PLos ONE 4:e4872.

66 Vogel, V. and Sheetz, M. (2009) Cell fate regulation by coupling mechanical cycles to biochemical signaling pathways. Current Opinion Cell Biol. 21:1-9.

67 Huang, S. (2009) Reprogramming cell fates: reconciling rarity with robustness. Bioessays 31:546-560.

68 Gatenby, R. A. and Frieden, B. R. (2007) Information theory in living systems, methods, applications and challenges. Bull. Math. Biol. 69:631-657.

69 Neves, S. (2008) Cell shape and negative links in regulatory motifs together control spatial information flow in signaling networks. Cell 133:666-680.

70 Perelson, A. S. and Bell, G. I. (1982) Delivery of lethal hits by cytotoxic T lymphocytes in multicellular conjugates occurs sequentially but at random times. J. Immunol. 129, 2796-2801.

71 Malawista, S. E. et al. (2006) Cryopreservable neutrophil surrogates: granule-poor, motile cytoplasts from polymorphonuclear leukocytes home to inflammatory lesions in vivo. Cell Motility Cytoskeleton 63, 254-257.

$72 \mathrm{Na}$, S. et al. (2008) Rapid signal transduction in living cells is a unique feature of mechanotransduction. Proc. Natl. Acad. Sci. USA 105, 6626-6631.

73 McKeithan, T. W. (1995) Kinetic proofreading in T-cell receptor signal transduction. Proc. Natl. Acad. Sci. 92, 5042-5046.

74 Salazar, C. et al. (2008) Decoding of calcium oscillations by phosphorylation cycles : analytic results. Biophys. J. 94, 1209-1215.

75 Nevozhay, D. et al. (2009) Negative autoregulation linearizes the dose-response and suppresses the heterogeneity of gene expression. Proc. Natl. Acad. Sci. USA 31:5123-5128.

76 Pecreaux, J. et al. (2004) Refined contour analysis of giant unilamellar vesicles. Eur. Phys. J. E 13, 277-290.

77 Helfrich, W. and Servuss, R. M. (1984) Undulations, steric interaction and cohesion of fluid membranes. Nuovo Cimento 3D, 137-151.

78 Robert, P. et al. (2007) what is the biological relevance of the specific bond properties revealed by single molecule studies? J. Mol. Recognition 20, 432-447.

79 Marshall, B. T. et al. (2005) Force history dependence of receptor-ligand dissociation. Biophys. J. 88:14581466.

80 Pincet, F. and Husson, J. (2005) The solution to the streptavidin-biotin paradox: the influence of history on the strength of single molecular bonds. Biophys. J. 89:4374-4381. 